\title{
MULTIPLICATIVE STRUCTURES FOR KOSZUL ALGEBRAS
}

\author{
RAGNAR-OLAF BUCHWEITZ, EDWARD L. GREEN ${ }^{\dagger}$, NICOLE SNASHALL, \\ AND ØYVIND SOLBERG ${ }^{\ddagger}$
}

\begin{abstract}
Let $\Lambda=k Q / I$ be a Koszul algebra over a field $k$, where $Q$ is a finite quiver. An algorithmic method for finding a minimal projective resolution $\mathbb{F}$ of the graded simple modules over $\Lambda$ is given in [9]. This resolution is shown to have a "comultiplicative" structure in [7], and this is used to find a minimal projective resolution $\mathbb{P}$ of $\Lambda$ over the enveloping algebra $\Lambda^{e}$. Using these results we show that the multiplication in the Hochschild cohomology ring of $\Lambda$ relative to the resolution $\mathbb{P}$ is given as a cup product and also provide a description of this product. This comultiplicative structure also yields the structure constants of the Koszul dual of $\Lambda$ with respect to a canonical basis over $k$ associated to the resolution $\mathbb{F}$. The natural map from the Hochschild cohomology to the Koszul dual of $\Lambda$ is shown to be surjective onto the graded centre of the Koszul dual.
\end{abstract}

\section{INTRODUCTION}

The aim of this paper is to show that the knowledge of a minimal projective resolution of the graded simple modules for a Koszul algebra over a field $k$ not only provides a $k$-basis for the Koszul dual with structure constants, but also gives a closed formula for the multiplication in the Hochschild cohomology ring. In a forthcoming paper [9] an algorithm will be presented for computing a minimal projective resolution of linear modules for a Koszul algebra, and thus making Koszul algebras more computationally accessible.

A prime example of a Koszul algebra is a polynomial ring in a finite number of commuting variables. Its Koszul dual is the exterior algebra, which is a finite dimensional selfinjective algebra. Using techniques from representation theory of finite dimensional algebras and Koszul duality, Martínez-Villa and Zacharia have obtained new results on the structure of locally free sheaves on projective space [13]. Polynomial rings are Artin-Schelter regular Koszul algebras and their Koszul duals are finite dimensional selfinjective algebras. More generally, this connection between representation theory of finite dimensional algebras and Artin-Schelter regular Koszul algebras allows one to apply the theory of support varieties for finite dimensional selfinjective algebras defined via the Hochschild cohomology ring (see $[4,16])$. To apply the theory of support varieties some finiteness conditions must be satisfied. These conditions are related to the natural map from the Hochschild cohomology ring to the Koszul dual. The image of this map is characterized in Section 4 of this paper.

Date: October 15, 2018.

2000 Mathematics Subject Classification. Primary: 16S37, 16 E40.

${ }^{\dagger}$ Partially supported by a grant from the NSA.

$\ddagger$ Partially supported by the Research Council of Norway. 
We now introduce notation we use throughout the paper and describe the results of the paper in more detail. Let $Q$ be a finite quiver and $k$ a field. We denote the ideal generated by the arrows in $Q$ in the path algebra $k Q$ by $J$. Let $\Lambda=k Q / I$ be a Koszul algebra, where $I$ is an ideal contained in $J^{2}$. Set $\mathfrak{r}=J / I$. In [10] a method for constructing a minimal projective resolution of $\Lambda / \mathfrak{r}$ as a right $\Lambda$-module was presented. If $R=k Q$, then this minimal right resolution of the degree zero part $\Lambda_{0}$ of $\Lambda$ can be given in terms of a filtration of right ideals

$$
\cdots \subseteq \amalg_{i=0}^{t_{n}} f_{i}^{n} R \subseteq \amalg_{i=0}^{t_{n-1}} f_{i}^{n-1} R \subseteq \cdots \subseteq \amalg_{i=0}^{t_{1}} f_{i}^{1} R \subseteq \amalg_{i=0}^{t_{0}} f_{i}^{0} R=R
$$

in $R$. In [7] it is shown that there is a comultiplicative structure associated to the $\left\{f_{i}^{n}\right\}$; in particular there exist $c_{p q}(n, i, r)$ in $k$ such that

$$
f_{i}^{n}=\sum_{p=0}^{t_{r}} \sum_{q=0}^{t_{n-r}} c_{p q}(n, i, r) f_{p}^{r} f_{q}^{n-r}
$$

for all $n \geq 1$, all $i$ in $\left\{0,1, \ldots, t_{n}\right\}$ and all $r$ in $\{0,1, \ldots, n\}$.

The paper begins with recalling a reduced bar resolution introduced in [2]. As in the standard setup [5], there is an associated cup product, which we show is the same as the Yoneda product in the Hochschild cohomology ring. This reduced bar resolution together with the comultiplicative structure is shown to provide a description of the multiplication in the Hochschild cohomology ring $\operatorname{HH}^{*}(\Lambda)$ of $\Lambda$ in Section 2. Recall that the enveloping algebra $\Lambda^{e}$ of $\Lambda$ is given by $\Lambda^{\mathrm{op}} \otimes_{k} \Lambda$. In our description of the multiplicative structure of $\mathrm{HH}^{*}(\Lambda)$, we use the minimal projective resolution of $\Lambda$ as a right $\Lambda^{e}$-module presented in [7].

Recall that an element $x$ in $k Q$ is called uniform if $x$ is non-zero and there exist vertices $u$ and $v$ in $Q$ such that $x=u x v$. If $x$ is a uniform element with $x=u x v$ with $u$ and $v$ vertices, then we write $\mathfrak{o}(x)=u$ and $\mathfrak{t}(x)=v$. It was shown in [10] that the $\left\{f_{i}^{n}\right\}$ can be chosen to be uniform elements. The projective modules occurring in a minimal projective resolution of $\Lambda$ over $\Lambda^{e}$ are given by $P^{n}=\amalg_{i=0}^{t_{n}} \Lambda \mathfrak{o}\left(f_{i}^{n}\right) \otimes_{k} \mathfrak{t}\left(f_{i}^{n}\right) \Lambda$ for $n \geq 0$ (see [11]). In Section 2 the multiplicative structure of the Hochschild cohomology ring of a Koszul algebra $\Lambda$ is shown to have the following description.

Theorem. Suppose that $\eta: P^{n} \rightarrow \Lambda$ and $\theta: P^{m} \rightarrow \Lambda$ represent elements in $\mathrm{HH}^{*}(\Lambda)$ and are given by $\eta\left(\mathfrak{o}\left(f_{i}^{n}\right) \otimes \mathfrak{t}\left(f_{i}^{n}\right)\right)=\lambda_{i}$ for $i=0,1, \ldots, t_{n}$ and $\theta\left(\mathfrak{o}\left(f_{i}^{m}\right) \otimes \mathfrak{t}\left(f_{i}^{m}\right)\right)=\lambda_{i}^{\prime}$ for $i=0,1, \ldots, t_{m}$. Then

$$
\eta * \theta\left(\mathfrak{o}\left(f_{i}^{n+m}\right) \otimes \mathfrak{t}\left(f_{i}^{n+m}\right)\right)=\sum_{p=0}^{t_{n}} \sum_{q=0}^{t_{m}} c_{p q}(n+m, i, n) \lambda_{p} \lambda_{q}^{\prime}
$$

for $i=0,1, \ldots, t_{m+n}$, where $\eta * \theta$ represents the product of $\eta$ and $\theta$ in $\mathrm{HH}^{*}(\Lambda)$.

The multiplicative structure of the Hochschild cohomology ring is not known for most algebras, including Koszul algebras. One class of Koszul algebras for which the product is known is radical square zero algebras $\Lambda=k Q / J^{2}$ with $Q$ not an oriented cycle; then all products of elements in $\mathrm{HH}^{\geq 1}(\Lambda)$ are zero by [3]. Another class of Koszul algebras where the ring structure has been determined can be found in [1], where, for algebras in this class, $\operatorname{HH}^{*}(\Lambda)$ is a finitely generated algebra.

Since $\Lambda$ is a Koszul algebra, its Koszul dual, $E(\Lambda)=\operatorname{Ext}_{\Lambda}^{*}(\Lambda / \mathfrak{r}, \Lambda / \mathfrak{r})$, is also a Koszul algebra. In Section 3 we show that the comultiplicative structure of the sets $\left\{f_{i}^{n}\right\}$ can be used to construct the structure constants of the Koszul dual of $\Lambda$ for a 
basis associated to the sets $\left\{f_{i}^{n}\right\}$. In particular, given the sets $\left\{f_{i}^{n}\right\}$, we construct a basis $\left\{\widehat{f}_{i}^{n}\right\}$ of $E(\Lambda)$ so that the product is given by

$$
\widehat{f}_{i}^{m} \widehat{f}_{j}^{n}=\sum_{l=0}^{t_{m+n}} c_{j i}(m+n, l, n) \widehat{f}_{l}^{m+n} .
$$

See Theorem 3.1 for details.

There is a natural map $\varphi_{\Lambda / \mathfrak{r}}: H^{*}(\Lambda) \rightarrow E(\Lambda)$ whose image is contained in the graded centre of $E(\Lambda)$. Recall that the graded centre $Z_{\mathrm{gr}}(E(\Lambda))$ is the subring of $E(\Lambda)$ generated by all homogeneous elements $z$ such that $z y=(-1)^{|z||y|} y z$ for each homogeneous element $y$ in $E(\Lambda)$, where $|x|$ denotes the degree of a homogeneous element $x$. In Section 4 we apply the results of the previous sections to show that $\varphi_{\Lambda / \mathrm{r}}$ has image $Z_{\mathrm{gr}}(E(\Lambda))$.

The paper ends with a number of examples illustrating our results.

Throughout, we consider right modules unless otherwise explicitly stated.

\section{A REDUCED BAR RESOlution}

For this section we fix the following notation and assumptions. Let $\Lambda$ be an algebra over a field $k$, and let $\mathfrak{a}$ be an ideal in $\Lambda$. Set $\Lambda_{0}=\Lambda / \mathfrak{a}$ and assume that the natural algebra homomorphism $\Lambda \rightarrow \Lambda_{0}$ is a split $k$-algebra homomorphism. Finally, assume that $\Lambda_{0}$ is a finite product of copies of $k$. For example, if $\Lambda=k Q / I$ with $I$ contained in $J^{2}$ and $\mathfrak{a}=J / I$, then $\Lambda$ satisfies these conditions.

In this section we recall a reduced bar resolution $(\mathbb{B}, d)$ of $\Lambda$ introduced in [2]. If $\Lambda_{0}=\prod_{i=1}^{m} k$, then we let $\left\{e_{1}, \ldots, e_{m}\right\}$ be a complete set of primitive orthogonal central idempotents of $\Lambda_{0}$. Define $(\mathbb{B}, d)$ by $B^{n}=\Lambda^{\otimes_{\Lambda_{0}}(n+2)}$, the $(n+2)$-fold tensor product of $\Lambda$ over $\Lambda_{0}$, and $d^{n}: B^{n} \rightarrow B^{n-1}$ by

$$
d^{n}\left(\lambda_{0} \otimes \cdots \otimes \lambda_{n+1}\right)=\sum_{i=0}^{n}(-1)^{i}\left(\lambda_{0} \otimes \cdots \otimes \lambda_{i} \lambda_{i+1} \otimes \cdots \otimes \lambda_{n+1}\right) .
$$

It is shown in [2, Lemma 1.1] that $(\mathbb{B}, d)$ is a projective $\Lambda^{e}$-resolution of $\Lambda$. When $\Lambda_{0}=k$, this is the usual bar resolution. Note that if $\Lambda_{0}=\prod_{i=1}^{m} k$ for $m>1$, then $\Lambda_{0}$ is not central in general, so that $\Lambda$ is not necessarily an algebra over $\Lambda_{0}$.

For the bar resolution $(\mathbb{B}, d)$ there is a chain map $\Delta: \mathbb{B} \rightarrow \mathbb{B} \otimes_{\Lambda} \mathbb{B}$ given by

$$
\Delta\left(\lambda_{0} \otimes \cdots \otimes \lambda_{n+1}\right)=\sum_{i=0}^{n}\left(\lambda_{0} \otimes \cdots \otimes \lambda_{i} \otimes 1\right) \otimes\left(1 \otimes \lambda_{i+1} \otimes \cdots \otimes \lambda_{n+1}\right)
$$

(for example see [15, 1.2]). If $\eta$ and $\theta$ in $\mathrm{HH}^{n}(\Lambda)$ and $\mathrm{HH}^{m}(\Lambda)$, respectively, are represented by $\eta: B^{n} \rightarrow \Lambda$ and $\theta: B^{m} \rightarrow \Lambda$, then the cup product $\eta \cup \theta$ in $\mathrm{HH}^{n+m}(\Lambda)$ is given by the following composition of maps

$$
\mathbb{B} \stackrel{\Delta}{\rightarrow} \mathbb{B} \otimes_{\Lambda} \mathbb{B} \stackrel{\eta \otimes \theta}{\longrightarrow} \Lambda \otimes_{\Lambda} \Lambda \stackrel{\nu}{\rightarrow} \Lambda,
$$

where $\nu: \Lambda \otimes_{\Lambda} \Lambda \rightarrow \Lambda$ is the multiplication map. We see that the cup product is

$$
\eta \cup \theta\left(\left(\lambda_{0} \otimes \cdots \otimes \lambda_{n+m+1}\right)\right)=\eta\left(\lambda_{0} \otimes \cdots \otimes \lambda_{n} \otimes 1\right) \theta\left(1 \otimes \lambda_{n+1} \otimes \cdots \otimes \lambda_{n+m+1}\right) .
$$

In [17] it is shown that any projective $\Lambda^{e}$-resolution $\mathbb{X}$ of $\Lambda$ gives rise to a "cup product", which coincides with the ordinary cup product. Let $\mathbb{X}$ be a projective $\Lambda^{e}$-resolution of $\Lambda$. There exists a chain map $D: \mathbb{X} \rightarrow \mathbb{X} \otimes_{\Lambda} \mathbb{X}$ lifting the identity, 
which is unique up to homotopy. Siegel and Witherspoon define a cup product of two elements $\eta$ in $\mathrm{HH}^{n}(\Lambda)$ and $\theta$ in $\mathrm{HH}^{m}(\Lambda)$ as above using the composition

$$
\mathbb{X} \stackrel{D}{\rightarrow} \mathbb{X} \otimes_{\Lambda} \mathbb{X} \stackrel{\eta \otimes \theta}{\rightarrow} \Lambda \otimes_{\Lambda} \Lambda \stackrel{\nu}{\rightarrow} \Lambda,
$$

and note that it is independent of the projective resolution $\mathbb{X}$ of $\Lambda$ and the chain map $D$. In Section 2 we give an explicit formula for $D$ for the minimal projective $\Lambda^{e}$-resolution of $\Lambda$ constructed here.

In [5] it is shown that the cup product and the Yoneda product coincide. We give a proof of this below. Let $\eta * \theta$ denote the Yoneda product of $\eta$ and $\theta$ viewing $\mathrm{HH}^{*}(\Lambda)$ as $\operatorname{Ext}_{\Lambda^{e}}^{*}(\Lambda, \Lambda)$.

Proposition 1.1. Let $\eta$ and $\theta$ be in $\mathrm{HH}^{n}(\Lambda)$ and $\mathrm{HH}^{m}(\Lambda)$, respectively, and suppose that they are represented by $\eta: B^{n} \rightarrow \Lambda$ and $\theta: B^{m} \rightarrow \Lambda$. Then

$$
\eta * \theta=\eta \cup \theta
$$

In particular, the cup product and the Yoneda product on $\mathrm{HH}^{*}(\Lambda)$ coincide.

Proof. Let $\eta$ and $\theta$ be in $\mathrm{HH}^{n}(\Lambda)$ and $\mathrm{HH}^{m}(\Lambda)$ respectively. Then $\eta$ and $\theta$ can be represented as $\Lambda^{e}$-maps $\eta: B^{n} \rightarrow \Lambda$ and $\theta: B^{m} \rightarrow \Lambda$. The Yoneda product $\eta * \theta$ is given via constructing a lifting of $\theta$ to a chain map $\widetilde{\theta}=\left\{\theta^{i}\right\}_{i}: \mathbb{B} \rightarrow \mathbb{B}[n]$, where $\mathbb{B}[n]$ denotes the degree $n$ shift of the complex $\mathbb{B}$. Define $\widetilde{\theta}$ to be the composition

$$
\mathbb{B} \stackrel{\Delta}{\rightarrow} \mathbb{B} \otimes_{\Lambda} \mathbb{B} \stackrel{\mathbb{B} \otimes \theta}{\longrightarrow} \mathbb{B} \otimes_{\Lambda} \Lambda[n] \stackrel{\nu}{\rightarrow} \mathbb{B}[n]
$$

It is clear from the definition of $\tilde{\theta}$ that it is a morphism of complexes. Furthermore, direct computations shows that $\widetilde{\theta}$ is a lifting of $\theta$ to a chain map. Immediately from the definition of $\widetilde{\theta}$ we infer that

$$
\begin{aligned}
\eta * \theta & =\eta \widetilde{\theta} \quad=\eta \nu(\mathbb{B} \otimes \theta) \Delta \\
& =\nu(\eta \otimes \Lambda[n])(\mathbb{B} \otimes \theta) \Delta=\nu(\eta \otimes \theta) \Delta \\
& =\eta \cup \theta .
\end{aligned}
$$

\section{The multiplicative Structure of the Hochschild COHOMOlOGy Ring}

In this section the multiplicative structure of the Hochschild cohomology ring of a Koszul algebra, $\Lambda=k Q / I$, is found using the comultiplicative structure of a minimal projective resolution of $\Lambda_{0}$ as a right $\Lambda$-module as given in equation (1) in the introduction. More precisely, given two homogeneous elements in the Hochschild cohomology ring, we give a closed formula for their product as a map from the appropriate projective module in a projective resolution of $\Lambda$ over $\Lambda^{e}$ to $\Lambda$. Note that this map may be non-zero, but represent zero in Hochschild cohomology, since the residue class of the map in Hochschild cohomology still needs to be computed (see Example 5.1). The crucial ingredient in the proof is the minimal projective resolution of $\Lambda$ as a right $\Lambda^{e}$-module constructed in [7] and the inclusion of this resolution into the reduced bar resolution.

We begin by recalling definitions, notation, and results that we need in this section. In this section $\Lambda=k Q / I$ denotes a Koszul algebra over some field $k$, where $Q$ is a finite quiver and $I \subseteq J^{2}$. Here $J$ denotes the ideal in $k Q$ generated by the arrows, and let $\Lambda_{0}=\Lambda /(J / I)$. If $M$ is a $k Q$-module and $m$ is in $M$, then $\bar{m}$ denotes the natural residue class of $m$ in $M / M I$. 
In [7], it was shown that a minimal projective resolution $(\mathbb{P}, \delta)$ of $\Lambda$ as a right $\Lambda^{e}$-module can be constructed from knowledge of a minimal projective resolution of $\Lambda_{0}$ over $\Lambda$ as a right $\Lambda$-module. Since we need the results from [7], we summarize them below.

A minimal projective resolution $(\mathbb{F}, d)$ of $\Lambda_{0}$ as a right $\Lambda$-module can be constructed from a sequence of right ideals in $R=k Q$ as follows: There exists a choice of integers $t_{n}$ in $\mathbb{Z}$ and of uniform elements $\left\{f_{i}^{n}\right\}_{i=0}^{t_{n}}$ in $R$, for all $n \geq 0$, with

$$
\cdots \subseteq \amalg_{i=0}^{t_{n}} f_{i}^{n} R \subseteq \amalg_{i=0}^{t_{n-1}} f_{i}^{n-1} R \subseteq \cdots \subseteq \amalg_{i=0}^{t_{1}} f_{i}^{1} R \subseteq \amalg_{i=0}^{t_{0}} f_{i}^{0} R=R
$$

such that $F^{n}=\amalg_{i=0}^{n} f_{i}^{n} R / \amalg_{i=0}^{n} f_{i}^{n} I$ for all $n \geq 0$ and $d^{n}: F^{n} \rightarrow F^{n-1}$ is induced by the inclusion $\amalg_{i=0}^{n} f_{i}^{n} R \hookrightarrow \amalg_{i=0}^{n-1} f_{i}^{n-1} R$ (see [10]). We choose $\left\{f_{i}^{0}\right\}_{i=0}^{t_{0}}$ to be the set of the vertices in $Q$ and $\left\{f_{i}^{1}\right\}_{i=0}^{t_{1}}$ to be the set of the arrows in $Q$. Moreover $\left\{f_{i}^{2}\right\}_{i=0}^{t_{2}}$ is a minimal set of homogeneous generators of degree two for $I$.

When we have a minimal projective resolution $(\mathbb{F}, d)$ of $\Lambda_{0}$ as a right $\Lambda$-module obtained from a set $\left\{f_{i}^{n}\right\}$, we simply say that the set $\left\{f_{i}^{n}\right\}$ defines a minimal projective resolution.

It is shown in [7] that a minimal projective resolution $(\mathbb{P}, \delta)$ of $\Lambda$ over $\Lambda^{e}$ is given by $P^{n}=\amalg_{i=0}^{t_{n}} \Lambda \mathfrak{o}\left(f_{i}^{n}\right) \otimes_{k} \mathfrak{t}\left(f_{i}^{n}\right) \Lambda$ for $n \geq 0$, where the differential $\delta^{n}: P^{n} \rightarrow$ $P^{n-1}$ is now described for $n \geq 1$ : In [7] it was shown that the sets $\left\{f_{i}^{n}\right\}$ have a "comultiplicative structure"; namely, there are elements $c_{p q}(n, i, r)$ in $k$ such that

$$
f_{i}^{n}=\sum_{p=0}^{t_{r}} \sum_{q=0}^{t_{n-r}} c_{p q}(n, i, r) f_{p}^{r} f_{q}^{n-r}
$$

for all $n \geq 1$, all $i$ in $\left\{0,1, \ldots, t_{n}\right\}$ and all $r$ in $\{0,1, \ldots, n\}$. To simplify notation, we sometimes write $\sum_{p, q}$ for $\sum_{p=0}^{s} \sum_{q=0}^{s^{\prime}}$ when the bounds of $p$ and $q$ are clear. For $n \geq 0$ and $i$ with $0 \leq i \leq t_{n}$ define $\varepsilon_{i}^{n}=(0, \ldots, 0, \underbrace{\mathfrak{o}\left(f_{i}^{n}\right) \otimes_{k} \mathfrak{t}\left(f_{i}^{n}\right)}_{i \text {-th component }}, 0, \ldots, 0)$ in $P^{n}$. Then the differential $\delta^{n}: P^{n} \rightarrow P^{n-1}$ is given by

$$
\delta^{n}\left(\varepsilon_{i}^{n}\right)=\sum_{j=0}^{t_{n-1}}\left(\sum_{p=0}^{t_{1}} c_{p j}(n, i, 1) \overline{f_{p}^{1}} \varepsilon_{j}^{n-1}+(-1)^{n} \sum_{q=0}^{t_{1}} c_{j q}(n, i, n-1) \varepsilon_{j}^{n-1} \overline{f_{q}^{1}}\right)
$$

for $i=0,1, \ldots, t_{n}$ and $n \geq 1$, and $\delta^{0}: \amalg_{i=0}^{t_{0}} \Lambda e_{i} \otimes_{k} e_{i} \Lambda \rightarrow \Lambda$ is the multiplication map. Note that $\overline{f_{j}^{n}}$ denotes the residue class of $f_{j}^{n}$ in $\amalg_{i=0}^{t_{n}} f_{i}^{n} R / \amalg_{i=0}^{t_{n}} f_{i}^{n} I$.

Recall that the reduced bar resolution $(\mathbb{B}, d)$ of $\Lambda$ over $\Lambda^{e}$ from the previous section is given by $B^{n}=\Lambda^{\otimes_{\Lambda_{0}}(n+2)}$ and the differential $d^{n}: B^{n} \rightarrow B^{n-1}$ is given by

$$
\begin{aligned}
& d^{n}\left(\lambda_{0} \otimes \lambda_{1} \otimes \cdots \otimes \lambda_{n+1}\right)= \\
& \quad \sum_{i=0}^{n}(-1)^{i}\left(\lambda_{0} \otimes \lambda_{1} \otimes \cdots \otimes \lambda_{i-1} \otimes \lambda_{i} \lambda_{i+1} \otimes \lambda_{i+2} \otimes \cdots \otimes \lambda_{n+1}\right) .
\end{aligned}
$$

Define $\partial_{i}: B^{n} \rightarrow B^{n-1}$ by

$$
\partial_{i}\left(\lambda_{0} \otimes \cdots \otimes \lambda_{n+1}\right)=\lambda_{0} \otimes \cdots \otimes \lambda_{i-1} \otimes \lambda_{i} \lambda_{i+1} \otimes \lambda_{i+2} \otimes \cdots \otimes \lambda_{n+1}
$$

for $i=0,1, \ldots, n$. Then $d^{n}=\sum_{i=0}^{n}(-1)^{i} \partial_{i}$.

Identify $\Lambda_{0}$ with the subalgebra of $\Lambda$ generated by the vertices. View $R$ as the tensor algebra $T_{\Lambda_{0}}(V)$, where $V$ is the $\Lambda_{0}$-bimodule generated by the arrows in $Q$. 
Since $\Lambda$ is a Koszul algebra, each $f_{i}^{n}$ is a linear combination of paths of length $n$. Hence each $f_{i}^{n}$ can be viewed uniquely as an element in $V^{\otimes_{\Lambda_{0}}{ }^{n}}$ for all $n$ and $i$; see the definition of $\psi\left(f_{i}^{n}\right)$ in the proposition below. Now we show that this enables us to find a natural embedding of $\mathbb{P}$ as a subcomplex of $\mathbb{B}$.

Proposition 2.1. Define $\mu_{n}: P^{n} \rightarrow B^{n}$ by

$$
\mu_{n}\left(\varepsilon_{i}^{n}\right)=1 \otimes_{\Lambda_{0}} \psi\left(f_{i}^{n}\right) \otimes_{\Lambda_{0}} 1
$$

where

$$
\psi\left(f_{i}^{n}\right)=\sum c_{i j_{1} j_{2} \ldots j_{n}} \overline{f_{j_{1}}^{1}} \otimes \cdots \otimes \overline{f_{j_{n}}^{1}}
$$

when $f_{i}^{n}=\sum c_{i j_{1} j_{2} \ldots j_{n}} f_{j_{1}}^{1} \cdots f_{j_{n}}^{1}$.

Then $\left\{\mu_{n}\right\}_{n \geq 0}$ is a chain map from $(\mathbb{P}, \delta)$ to $(\mathbb{B}, d)$.

Proof. If $f_{i}^{n}=\sum_{p, q} c_{p q}(n, i, r) f_{p}^{r} f_{q}^{n-r}$, then $\psi\left(f_{i}^{n}\right)=\sum_{p, q} c_{p q}(n, i, r) \psi\left(f_{p}^{r}\right) \otimes \psi\left(f_{q}^{n-r}\right)$.

We have that

$$
\begin{aligned}
f_{i}^{n} & =\sum_{p, q} c_{p q}(n, i, r) f_{p}^{r} f_{q}^{n-r} \\
& =\sum_{p, q} c_{p q}(n, i, r) f_{p}^{r}\left(\sum_{p^{\prime}, q^{\prime}} c_{p^{\prime} q^{\prime}}(n-r, q, 2) f_{p^{\prime}}^{2} f_{q^{\prime}}^{n-r-2}\right)
\end{aligned}
$$

for any $r$ with $0 \leq r \leq n$. It follows from this that

$$
\partial_{r} \mu_{n}\left(\varepsilon_{i}^{n}\right)=\sum_{p, q} c_{p q}(n, i, r) \otimes \psi\left(f_{p}^{r}\right) \otimes\left(\sum_{p^{\prime}, q^{\prime}} c_{p^{\prime} q^{\prime}}(n-r, q, 2) \overline{f_{p^{\prime}}^{2}} \otimes \psi\left(f_{q^{\prime}}^{n-r-2}\right)\right) \otimes 1
$$

whenever $n \geq 2$ and $0 \leq r \leq n-2$. We see that $\partial_{r} \mu_{n}\left(\varepsilon_{i}^{n}\right)$ is zero since $f_{i_{p^{\prime}}}^{2}$ is in the ideal $I$ for all $p^{\prime}$. We infer from this that

$$
\begin{aligned}
d^{n} \mu_{n}\left(\varepsilon_{i}^{n}\right)=\sum_{p, q} c_{p q}(n, i, 1) \overline{f_{p}^{1}} \otimes & \psi\left(f_{q}^{n-1}\right) \otimes 1 \\
& +(-1)^{n} \sum_{p^{\prime}, q^{\prime}} c_{p^{\prime} q^{\prime}}(n, i, n-1) \otimes \psi\left(f_{p^{\prime}}^{n-1}\right) \otimes \overline{f_{q^{\prime}}^{1}}
\end{aligned}
$$

and consequently that $d^{n} \mu_{n}=\mu_{n-1} \delta^{n}$. This shows that the chain map $\left\{\mu_{n}\right\}_{n \geq 0}$ defines $(\mathbb{P}, \delta)$ as a subcomplex of the reduced bar resolution $(\mathbb{B}, d)$ from the previous section.

A projective resolution of $\Lambda$ over $\Lambda^{e}$ different from the bar resolution was first described for a Koszul algebra $\Lambda$ in $\left[14\right.$, Section 3.7] (choose $R=L=A$ in $K_{*}(R, A, L)$ in the notation of [14]). Furthermore, an embedding of this resolution into the bar resolution is given in [14, Proposition 3.9].

Given two homogeneous elements $\eta$ and $\theta$ in the cohomology of $\operatorname{Hom}_{\Lambda^{e}}(\mathbb{B}, \Lambda)$ their product $\eta * \theta$ in $\operatorname{HH}^{*}(\Lambda)$ is given as the composition of the maps

$$
\mathbb{B} \stackrel{\Delta}{\longrightarrow} \mathbb{B} \otimes_{\Lambda} \mathbb{B} \stackrel{\eta \otimes \theta}{\longrightarrow} \Lambda \otimes_{\Lambda} \Lambda \stackrel{\nu}{\rightarrow} \Lambda \text {. }
$$

Let $\mu: \mathbb{P} \rightarrow \mathbb{B}$ be the inclusion found above, and let $\pi: \mathbb{B} \rightarrow \mathbb{P}$ be a chain map such that $\pi \mu=\mathrm{id}_{\mathbb{P}}$.

Suppose that the image $\Delta(\mu \mathbb{P})$ is contained in $\mu \mathbb{P} \otimes_{\Lambda} \mu \mathbb{P} \subseteq \mathbb{B} \otimes_{\Lambda} \mathbb{B}$. In that case, $\Delta$ induces a map $\mathbb{P} \rightarrow \mathbb{P} \otimes_{\Lambda} \mathbb{P}$ that we denote by $\Delta^{\prime}$. In particular $\Delta \mu=(\mu \otimes \mu) \Delta^{\prime}$. By the results from [17] mentioned prior to Proposition 1.1, the cup product defined by $\Delta^{\prime}$ gives the multiplication in $\operatorname{HH}^{*}(\Lambda)$. 
Next we show that this is indeed the case; that is, $\Delta(\mu \mathbb{P})$ is contained in $\mu \mathbb{P} \otimes_{\Lambda} \mu \mathbb{P}$. To this end define $\Delta^{\prime}: \mathbb{P} \rightarrow \mathbb{P} \otimes_{\Lambda} \mathbb{P}$ by

$$
\Delta^{\prime}\left(\varepsilon_{i}^{n}\right)=\sum_{r=0}^{n} \sum_{p=0}^{t_{r}} \sum_{q=0}^{t_{n-r}} c_{p q}(n, i, r) \varepsilon_{p}^{r} \otimes_{\Lambda} \varepsilon_{q}^{n-r} .
$$

Proposition 2.2. Let $\Delta$ and $\Delta^{\prime}$ be as above. Then

$$
\Delta \mu=(\mu \otimes \mu) \Delta^{\prime}
$$

Proof. Let $s_{r}: \mathbb{B} \rightarrow \mathbb{B} \otimes_{\Lambda} \mathbb{B}$ be given by

$$
s_{r}\left(\lambda_{0} \otimes \cdots \otimes \lambda_{n+1}\right)=\left(\lambda_{0} \otimes \cdots \otimes \lambda_{r} \otimes 1\right) \otimes_{\Lambda}\left(1 \otimes \lambda_{r+1} \otimes \cdots \otimes \lambda_{n+1}\right)
$$

for $r=0,1, \ldots, n$. Then we have that $\Delta=\sum_{r=0}^{n} s_{r}$. Furthermore,

$$
\begin{aligned}
s_{r}\left(\mu_{n}\left(\varepsilon_{i}^{n}\right)\right) & =\sum_{p, q}\left(c_{p q}(n, i, r) \otimes \psi\left(f_{p}^{r}\right) \otimes 1\right) \otimes_{\Lambda}\left(1 \otimes \psi\left(f_{q}^{n-r}\right) \otimes 1\right) \\
& =\sum_{p, q}\left(c_{p q}(n, i, r) \mu_{r}\left(\varepsilon_{p}^{r}\right) \otimes_{\Lambda} \mu_{n-r}\left(\varepsilon_{q}^{n-r}\right)\right. \\
& =\left(\mu_{r} \otimes \mu_{n-r}\right) \Delta^{\prime}\left(\varepsilon_{i}^{n}\right) .
\end{aligned}
$$

Since $\Delta=\sum_{r=0}^{n} s_{r}$, we have that $\Delta \mu_{n}\left(\varepsilon_{i}^{n}\right)=(\mu \otimes \mu) \Delta^{\prime}\left(\varepsilon_{i}^{n}\right)$. Hence the claim follows.

Combining the results above we obtain our result on the multiplicative structure for the Hochschild cohomology ring of a Koszul algebra.

Theorem 2.3. Let $\Lambda=k Q / I$ with $I \subseteq J^{2}$ be a Koszul algebra over a field $k$, where $Q$ is a finite quiver. Suppose that $\eta: P^{n} \rightarrow \Lambda$ and $\theta: P^{m} \rightarrow \Lambda$ represent elements in $\mathrm{HH}^{*}(\Lambda)$ and that they are given by $\eta\left(\varepsilon_{i}^{n}\right)=\lambda_{i}$ for $i=0,1, \ldots, t_{n}$ and $\theta\left(\varepsilon_{i}^{m}\right)=\lambda_{i}^{\prime}$ for $i=0,1, \ldots, t_{m}$. Then

$$
(\eta * \theta)\left(\varepsilon_{i}^{m+n}\right)=\sum_{p=0}^{t_{n}} \sum_{q=0}^{t_{m}} c_{p q}(n+m, i, n) \lambda_{p} \lambda_{q}^{\prime}
$$

for all $i=0,1, \ldots, t_{m+n}$.

Proof. By Proposition 1.1, we have that $\eta * \theta=\eta \cup \theta$. Using the results from [17] reviewed prior to Proposition 1.1 we infer that

$$
\begin{aligned}
\eta * \theta\left(\varepsilon_{i}^{m+n}\right) & =\nu(\eta \otimes \theta) \Delta^{\prime}\left(\varepsilon_{i}^{m+n}\right) \\
& =\nu(\eta \otimes \theta)\left(\sum_{r=0}^{n+m} \sum_{p, q} c_{p q}(n+m, i, r) \varepsilon_{p}^{r} \otimes_{\Lambda} \varepsilon_{q}^{n-r}\right) \\
& =\sum_{p, q} c_{p q}(n+m, i, n) \eta\left(\varepsilon_{p}^{n}\right) \theta\left(\varepsilon_{q}^{m}\right) \\
& =\sum_{p, q} c_{p q}(n+m, i, n) \lambda_{p} \lambda_{q}^{\prime}
\end{aligned}
$$

This completes the proof of the theorem. 


\section{Structure constants for the Koszul Dual}

In this section we give a second application of the comultiplication formula (1). We show that, for a Koszul algebra $\Lambda=k Q / I$, equation (1) gives the structure constants for a basis for the Koszul dual of $\Lambda$. Of course the Koszul dual can immediately be given by generators and relations. But for computational purposes, it is sometimes important to find a $k$-basis and the structure constants for that basis.

Suppose that the sets $\left\{f_{i}^{n}\right\}$ define a minimal projective resolution $(\mathbb{F}, e)$ of $\Lambda / \mathfrak{r}$ as a right $\Lambda$-module. Note that $F^{n}=\amalg_{i=0}^{t_{n}} f^{n} R / \amalg_{i=0}^{t_{n}} f^{n} I$. Define $\widehat{f}_{i}^{n}: F^{n} \rightarrow \Lambda / \mathfrak{r}$ for $i=0,1, \ldots, t_{n}$ by $\widehat{f}_{i}^{n}\left(\overline{f_{j}^{n}}\right)=\delta_{i j} \mathfrak{t}\left(f_{i}^{n}\right)$ for all $j=0,1, \ldots, t_{n}$. Since $(\mathbb{F}, e)$ is a minimal projective resolution, it is immediate that the elements $\left\{\widehat{f}_{i}^{n}\right\}_{i=0}^{t_{n}}$ form a basis for $\operatorname{Ext}_{\Lambda}^{n}(\Lambda / \mathfrak{r}, \Lambda / \mathfrak{r})$ as a vector space over $k$. The goal of this section is to find the structure constants for this particular $k$-basis for the Koszul dual of $\Lambda$. We note that $\Lambda$ is given by generators and relations, whereas the multiplication in the Koszul dual is given by structure constants. Since $\Lambda$ is the Koszul dual of its Koszul dual, we may construct the set $\left\{f_{i}^{n}\right\}$ for the Koszul dual and then the results here can be applied to find a $k$-basis of $\Lambda$ and its structure constants.

Theorem 3.1. Let $\Lambda=k Q / I$ be a Koszul algebra over some field $k$, where $Q$ is a finite quiver and $I \subseteq J^{2}$. Denote by $\left\{f_{i}^{n}\right\}_{i=0}^{t_{n}}$ elements in $k Q$ defining a minimal projective resolution $(\mathbb{F}, e)$ of $\Lambda / \mathfrak{r}$ as a right module over $\Lambda$. Let the elements $\widehat{f}_{i}^{n}: F^{n} \rightarrow \Lambda / \mathfrak{r}$ for $i=0,1, \ldots, t_{n}$ be given by $\widehat{f}_{i}^{n}\left(\overline{f_{j}^{n}}\right)=\delta_{i j} \mathfrak{t}\left(f_{i}^{n}\right)$, for all $j=0,1, \ldots, t_{n}$. The set $\left\{\widehat{f}_{i}^{n}\right\}_{i=0}^{t_{n}}$ represents a $k$-basis of $\operatorname{Ext}_{\Lambda}^{n}(\Lambda / \mathfrak{r}, \Lambda / \mathfrak{r})$.

Then the product $\widehat{f}_{i}^{m} \widehat{f}_{j}^{n}$ in $\operatorname{Ext}_{\Lambda}^{*}(\Lambda / \mathfrak{r}, \Lambda / \mathfrak{r})$ of the two elements $\widehat{f}_{i}^{m}$ and $\widehat{f}_{j}^{n}$ in degree $m$ and $n$ in $\operatorname{Ext}_{\Lambda}^{*}(\Lambda / \mathfrak{r}, \Lambda / \mathfrak{r})$, respectively, is given by

$$
\widehat{f}_{i}^{m} \widehat{f}_{j}^{n}=\sum_{l=0}^{t_{m+n}} c_{j i}(m+n, l, n) \widehat{f}_{l}^{m+n} .
$$

Proof. A lifting of the map $\widehat{f}_{j}^{n}: F^{n} \rightarrow \Lambda / \mathfrak{r}$ to a map $g_{0}: F^{n} \rightarrow F^{0}$ is given by $g_{0}\left(\overline{f_{t}^{n}}\right)=\delta_{j t} \mathfrak{t}\left(f_{j}^{n}\right)$. We want to find a lifting of $g_{0}$ to a chain map $\mathbb{F}[n] \rightarrow \mathbb{F}$, where $\mathbb{F}[i]$ denotes the $i$-th shift of the complex $\mathbb{F}$. Define $g_{r}: F^{n+r} \rightarrow F^{r}$ by letting

$$
g_{r}\left(\overline{f_{i}^{n+r}}\right)=\sum_{q=0}^{t_{r}} \overline{f_{q}^{r}} c_{j q}(n+r, i, n)
$$

for $i=0,1, \ldots, t_{n+r}$. Then it is clear that $g_{0} e^{n+1}=e^{1} g_{1}$. To show that $g_{r} e^{n+r+1}=$ $e^{r+1} g_{r+1}$ for $r \geq 1$ consider the following equalities:

$$
\begin{aligned}
f_{l}^{n+r+1} & =\sum_{p=0}^{t_{n+r}} \sum_{q=0}^{t_{1}} f_{p}^{n+r} f_{q}^{1} c_{p q}(n+r+1, l, n+r) \\
& =\sum_{p=0}^{t_{n+r}} \sum_{q=0}^{t_{1}} \sum_{x=0}^{t_{n}} \sum_{y=0}^{t_{r}} f_{x}^{n} f_{y}^{r} f_{q}^{1} c_{x y}(n+r, p, n) c_{p q}(n+r+1, l, n+r)
\end{aligned}
$$


and

$$
\begin{aligned}
f_{l}^{n+r+1} & =\sum_{x=0}^{t_{n}} \sum_{v=0}^{t_{r+1}} f_{x}^{n} f_{v}^{r+1} c_{x v}(n+r+1, l, n) \\
& =\sum_{x=0}^{t_{n}} \sum_{v=0}^{t_{r+1}} \sum_{y=0}^{t_{r}} \sum_{q=0}^{t_{1}} f_{x}^{n} f_{y}^{r} f_{q}^{1} c_{x v}(n+r+1, l, n) c_{y q}(r+1, v, r) .
\end{aligned}
$$

Since $\sum_{x=0}^{t_{n}} f_{x}^{n} R$ is a direct sum, for $x=j$, we have that

$$
\begin{aligned}
& \sum_{p=0}^{t_{n+r}} \sum_{q=0}^{t_{1}} \sum_{y=0}^{t_{r}} f_{y}^{r} f_{q}^{1} c_{j y}(n+r, p, n) c_{p q}(n+r+1, l, n+r)= \\
& \sum_{v=0}^{t_{r+1}} \sum_{y=0}^{t_{r}} \sum_{q=0}^{t_{1}} f_{y}^{r} f_{q}^{1} c_{j v}(n+r+1, l, n) c_{y q}(r+1, v, r)
\end{aligned}
$$

as elements in $\amalg_{y=0}^{t_{r}} f_{y}^{r} R$. Taking these equalities in $\amalg_{y=0}^{t_{r}} f_{y}^{r} R / \amalg_{y=0}^{t_{r}} f_{y}^{r} I$, the left hand side of this equality is $g_{r} e^{n+r+1}\left(\overline{f_{l}^{n+r+1}}\right)$ and the right hand side is $e^{r+1} g_{r+1}\left(\overline{f_{l}^{n+r+1}}\right)$ for all $l=0,1, \ldots, t_{n+r+1}$, hence $g_{r} e^{n+r+1}=e^{r+1} g_{r+1}$. We have that $\widehat{f}_{i}^{m} \widehat{f}_{j}^{n}=\widehat{f}_{i}^{m} g_{m}$, so that $\widehat{f}_{i}^{m} \widehat{f}_{j}^{n}=\sum_{l=0}^{t_{m+n}} c_{j i}(m+n, l, n) \widehat{f}_{l}^{m+n}$. This completes the proof.

\section{The graded Centre And Hochschild COHOMOlOGy}

For a finite dimensional algebra or graded algebra $\Lambda=k Q / I$ the image of the natural map $\varphi_{\Lambda / \mathfrak{r}}: \operatorname{HH}^{*}(\Lambda) \rightarrow \operatorname{Ext}_{\Lambda}^{*}(\Lambda / \mathfrak{r}, \Lambda / \mathfrak{r})=E(\Lambda)$ is shown to be contained in the graded centre $Z_{\mathrm{gr}}(E(\Lambda))$ in [16]. Here $\varphi_{\Lambda / \mathfrak{r}}$ is induced from $\Lambda / \mathfrak{r} \otimes_{\Lambda}-:(\mathbb{P}, \delta) \rightarrow$ $\left(\Lambda / \mathfrak{r} \otimes_{\Lambda} \mathbb{P}, 1 \otimes \delta\right)$. It was independently observed by Buchweitz and Green-SnashallSolberg that when $\Lambda$ is a Koszul algebra, the image is in fact equal to $Z_{\mathrm{gr}}(E(\Lambda))$. This was obtained by Buchweitz as a part of a more general isomorphism between the Hochschild cohomology ring of $\Lambda$ and the graded Hochschild cohomology ring of the Koszul dual. This isomorphism has since been generalized by Keller [12]. We note that the image of $\varphi_{\Lambda / \mathfrak{r}}$ is in general strictly contained in $Z_{\mathrm{gr}}(E(\Lambda))$, see [8, Example 7.6]. In this section we give an elementary proof of the fact that the image is $Z_{\mathrm{gr}}(E(\Lambda))$ for a Koszul algebra $\Lambda$ using the results from the previous sections.

Theorem 4.1. Let $\Lambda=k Q / I$ be a Koszul algebra. The image of the natural map $\varphi_{\Lambda / \mathrm{r}}: \mathrm{HH}^{*}(\Lambda) \rightarrow E(\Lambda)$ is the graded centre $Z_{\mathrm{gr}}(E(\Lambda))$.

Proof. We keep the notation developed in the previous sections. Let $z=\sum_{i=0}^{t_{n}} \alpha_{i} \widehat{f}_{i}^{n}$ with $\alpha_{i}$ in $k$. Since $\Lambda$ is a Koszul algebra, $E(\Lambda)$ is generated in degrees 0 and 1 . Hence $z$ is in $Z_{\mathrm{gr}}(E(\Lambda))$ if and only if $(\mathrm{A}) \widehat{f}_{j}^{1} z=(-1)^{n} z \widehat{f}_{j}^{1}$ for all $j=0,1, \ldots, t_{1}$ and (B) $\widehat{f}_{l}^{0} z=z \widehat{f}_{l}^{0}$ for all $l=0,1, \ldots, t_{0}$. Using the structure constants found in Theorem 3.1, we have that

$$
\widehat{f}_{j}^{1} z=\sum_{i=0}^{t_{n}} \alpha_{i} \widehat{f}_{j}^{1} \widehat{f}_{i}^{n}=\sum_{i=0}^{t_{n}} \alpha_{i} \sum_{l=0}^{t_{n+1}} c_{i j}(n+1, l, n) \widehat{f}_{l}^{n+1}
$$


and

$$
z \widehat{f}_{j}^{1}=\sum_{i=0}^{t_{n}} \alpha_{i} \widehat{f}_{i}^{n} \widehat{f}_{j}^{1}=\sum_{i=0}^{t_{n}} \alpha_{i} \sum_{l=0}^{t_{n+1}} c_{j i}(n+1, l, 1) \widehat{f}_{l}^{n+1} .
$$

Suppose that $z$ is in $Z_{\mathrm{gr}}(E(\Lambda))$. Then the condition (B) above implies that $\mathfrak{o}\left(f_{i}^{n}\right)=$ $\mathfrak{t}\left(f_{i}^{n}\right)$ for all $i$ such that $\alpha_{i} \neq 0$ and

$$
\sum_{i=0}^{t_{n}} \alpha_{i} c_{i j}(n+1, l, n)=(-1)^{n} \sum_{i=0}^{t_{n}} \alpha_{i} c_{j i}(n+1, l, 1) .
$$

Define $\eta: P^{n} \rightarrow \Lambda$ by letting $\eta\left(\varepsilon_{i}^{n}\right)=\alpha_{i} \mathfrak{t}\left(f_{i}^{n}\right)$ for $i=0,1, \ldots, t_{n}$. Then

$$
\begin{aligned}
\eta \delta^{n+1}\left(\varepsilon_{l}^{n+1}\right) & =\sum_{i=0}^{t_{n}} \sum_{j=0}^{t_{1}} c_{j i}(n+1, l, 1) \overline{f_{j}^{1}} \alpha_{i}+(-1)^{n+1} \sum_{i=0}^{t_{n}} \sum_{q=0}^{t_{1}} c_{i q}(n+1, l, n) \overline{f_{q}^{1}} \alpha_{i} \\
& =\sum_{j=0}^{t_{1}} \sum_{i=0}^{t_{n}}\left(c_{j i}(n+1, l, 1)+(-1)^{n+1} c_{i j}(n+1, l, n)\right) \alpha_{i} \overline{f_{j}^{1}} \\
& =0 .
\end{aligned}
$$

for all $l=0,1, \ldots, t_{n+1}$ and $j=0,1, \ldots, t_{1}$. The last equality follows since $z$ is in $Z_{\mathrm{gr}}(E(\Lambda))$. Hence $\eta$ is in $\mathrm{HH}^{n}(\Lambda)$, and $\varphi_{\Lambda / \mathfrak{r}}(\eta)=z$. This completes the proof.

In [16] it is conjectured that, if $\Lambda$ is a finite dimensional algebra over a field $k$, then the Hochschild cohomology ring of $\Lambda$ modulo the ideal generated by the homogeneous nilpotent elements is a finitely generated commutative algebra over $k$. As a consequence of the previous result, for a finite dimensional Koszul algebra $\Lambda$, the conjecture is equivalent to the conjecture that $Z_{\mathrm{gr}}(E(\Lambda))$, modulo the ideal generated by homogeneous nilpotent elements, is a finitely generated algebra over $k$. This can be seen by noting that the kernel of $\varphi_{\Lambda / \mathrm{r}}$ is contained in the ideal generated by homogeneous nilpotent elements [16]. We apply these ideas in Example 5.3.

\section{EXAMPLES}

Example 5.1. This example shows that the multiplication formula in Theorem 2.3 only gives a representative of a product in Hochschild cohomology as a map from the appropriate projective to the Koszul algebra. Thus, for example, to find if two products are equal, the residue classes must be computed.

Let $\Lambda=k\langle x, y\rangle /\left(x^{2}, x y+y x\right)$ for a field $k$ of characteristic different from 2 . Since $\left\{x^{2}, x y+y x\right\}$ is a quadratic Gröbner basis for the ideal generated by the relations under the length lexicographic order with $x>y>1$, the algebra $\Lambda$ is Koszul [6]. In this example $t_{0}=0$, and $t_{n}=1$ for all $n \geq 1$. We have that $f_{0}^{0}=1$, and $f_{0}^{n}=x^{n}$ and $f_{1}^{n}=\sum_{\substack{a, b \geq 0 \\ a+b=n-1}} x^{a} y x^{b}$ for $n \geq 1$. Set $f_{1}^{0}=0$. For $n \geq 1$ and $r$ with $0 \leq r \leq n$, we see that $f_{0}^{n}=f_{0}^{r} f_{0}^{n-r}$ and that $f_{1}^{n}=f_{0}^{r} f_{1}^{n-r}+f_{1}^{r} f_{0}^{n-r}$. Hence, $c_{00}(n, 0, r)=c_{01}(n, 1, r)=c_{10}(n, 1, r)=1$ and all other $c_{p q}(n, i, r)=0$. In particular, $c_{01}(2,1,1)=c_{10}(2,1,1)=1$. Let $(\mathbb{P}, \delta)$ be the minimal projective resolution of $\Lambda$ as a right $\Lambda^{e}$-module described in Section 2. Then $P^{1}=\varepsilon_{0}^{1} \Lambda^{e} \amalg \varepsilon_{1}^{1} \Lambda^{e}$. Define $\eta: P^{1} \rightarrow \Lambda$ by $\eta\left(\varepsilon_{i}^{1}\right)=\left\{\begin{array}{ll}x y, & i=0 \\ y, & i=1\end{array}\right.$, and $\theta: P^{1} \rightarrow \Lambda$ by $\eta\left(\varepsilon_{i}^{1}\right)=\left\{\begin{array}{ll}0, & i=0 \\ y, & i=1\end{array}\right.$. The reader may check that both $\eta$ and $\theta$ represent non-zero elements in Hochschild 
cohomology. On the other hand, the map $\eta * \theta$ is non-zero, but represents zero in $\mathrm{HH}^{2}(\Lambda)$.

Example 5.2. Let $Q$ be a finite quiver, which is not an oriented cycle. Recall that $J$ denotes the ideal of $k Q$ generated by the arrows of $Q$. Let $\Lambda=k Q / J^{2}$, which is a Koszul algebra. Then $t_{n}+1$ is the number of paths of length $n$ for all $n \geq 0$, and we choose the set $\left\{f_{i}^{n}\right\}_{i=0}^{t_{n}}$ to be the set of all paths of length $n$ in some order. Given $n$ and $r$ with $0 \leq r \leq n$, and $i$ with $0 \leq i \leq t_{n}$, there exist unique $j$ and $l$ with $0 \leq j \leq t_{r}$ and $0 \leq l \leq t_{n-r}$ such that $f_{i}^{n}=f_{j}^{r} f_{l}^{n-r}$. Hence $c_{p q}(n, i, r)=\left\{\begin{array}{ll}1, & \text { if } p=j \text { and } q=l \\ 0, & \text { otherwise }\end{array}\right.$. A classic result states that the Koszul dual of $\Lambda$ is $k Q^{\text {op }}$, where $Q^{\text {op }}$ is the opposite quiver of $Q$. If we write $f_{i}^{n}=f_{j_{1}}^{1} \cdots f_{j_{n}}^{1}$ as a product of arrows, then by Theorem 3.1 the element $\widehat{f}_{i}^{n}=\widehat{f}_{j_{n}}^{1} \cdots \widehat{f}_{j_{1}}^{1}$ and therefore the Koszul dual of $\Lambda$ is isomorphic to $k Q^{\text {op }}$.

For a discussion of the Hochschild cohomology ring of $\Lambda$ we refer to [8, Lemma 7.4] and the remarks following it or to [3].

Example 5.3. Let $\Lambda=k\langle x, y, z\rangle /\left(x^{2}, y^{2}, z^{2}, x y+a y x, x z+b z x, y z+c z y\right)$ be the quantum exterior algebra over a field $k$ with $a, b$ and $c$ non-zero elements in $k$. This algebra is easily seen to be selfinjective of dimension 8 over $k$. In the length lexicographic order with $x>y>z>1$, the set $\left\{x^{2}, y^{2}, z^{2}, x y+a y x, x z+b z x, y z+\right.$ $c z y\}$ is a quadratic Gröbner basis for the ideal it generates, and hence $\Lambda$ is a Koszul algebra [6]. The Koszul dual of $\Lambda$ is quantum 3-space, $R=k\langle x, y, x\rangle /(y x-a x y, z x-$ $b x z, z y-c y z)$. Note that $R$ is Artin-Schelter regular of global dimension 3 .

The Hochschild cohomology ring of the algebra $\Sigma=k\langle x, y\rangle /\left(x^{2}, x y+q y x, y^{2}\right)$ for $q$ in $k$ was studied in detail in [1]. In that paper it was shown that when $q$ is not a root of unity, then the Hochschild cohomology groups $\mathrm{HH}^{n}(\Sigma)$ vanish for $n \geq 3$. In this example we show that a similar phenomena occurs for $\Lambda$. In particular, for certain values of $a, b$, and $c$, we show that $\operatorname{HH}^{n}(\Lambda)$ vanishes for $n \geq 4$.

For ease of notation, we triply index the set $\left\{f_{i}^{n}\right\}$ as the set $\left\{f_{u, v, w}^{n}\right\}$, where $u+v+w=n$. A choice of the elements $\left\{f_{u, v, w}^{n}\right\}$ defining a minimal resolution of $\Lambda / \mathfrak{r}$ as a right $\Lambda$-module can be given inductively as follows: $f_{0,0,0}^{0}=1$ and for non-negative integers $u, v$, and $w$ with $u+v+w=n$,

$$
f_{u, v, w}^{n}=a^{v} b^{w} f_{u-1, v, w}^{n-1} x+c^{w} f_{u, v-1, w}^{n-1} y+f_{u, v, w-1}^{n-1} z
$$

with the requirement that $f_{-1, v, w}^{n}=f_{u,-1, w}^{n}=f_{u, v,-1}^{n}=0$ and that $f_{u, v, w}^{n}=0$ for $u+v+w>n$. The reader may check that for each $n$, the number of $f_{u, v, w}^{n}$ 's is $\left(\begin{array}{c}n+2 \\ n\end{array}\right)$.

Let $(\mathbb{P}, \delta)$ be the minimal projective $\Lambda^{e}$-resolution of $\Lambda$ defined in Section 2 . To compute the Hochschild cohomology groups, we compute the dimension of the image of the map

$$
\left(\delta^{n}\right)^{*}=\operatorname{Hom}_{\Lambda^{e}}\left(\delta^{n}, \Lambda\right): \operatorname{Hom}_{\Lambda^{e}}\left(P^{n-1}, \Lambda\right) \rightarrow \operatorname{Hom}_{\Lambda^{e}}\left(P^{n}, \Lambda\right) .
$$

One finds that the dimension of $\operatorname{Im}\left(\delta^{n}\right)^{*}$ is given by $2 n^{2}+4 n+1$ when $n \geq 3$ if, for example, $a, b$ and $c$ are algebraically independent elements of $k$. This implies that the Hochschild cohomology groups $\operatorname{HH}^{n}(\Lambda)$ vanish for $n \geq 4$, when $a, b$ and $c$ are algebraically independent elements of $k$.

In the case when $a, b$ and $c$ are algebraically independent, it is easy to see that $Z_{\mathrm{gr}}(E(\Lambda))$ is just $k$. On the other hand when the characteristic of $k$ is not two, 
and if $a=b=1$ and $c$ is not a root of unity, then we see that $Z_{\mathrm{gr}}(E(\Lambda))=k\left[x^{2}\right]$ in $E(\Lambda)$. If $c$ is a primitive $m$-th root of unity, then $Z_{\mathrm{gr}}(E(\Lambda))=k\left[x^{2}, y^{m}, z^{m}\right]$ in $E(\Lambda)$. By the result of Section 4, we see that the non-nilpotent elements of $Z_{\mathrm{gr}}(E(\Lambda))$ correspond to non-nilpotent elements of $\mathrm{HH}^{*}(\Lambda)$. Hence the Hochschild cohomology groups $\mathrm{HH}^{n}(\Lambda)$ are non-zero in all even degrees for these choices of $a$, $b$, and $c$.

We leave it to the reader to check that

$$
f_{u, v, w}^{n}=x f_{u-1, v, w}^{n-1}+a^{u} y f_{u, v-1, t}^{n-1}+b^{u} c^{v} z f_{u, v, w-1}^{n-1} .
$$

Furthermore, for $r$ with $0 \leq r \leq n$,

$f_{u, v, w}^{n}=\sum_{s=\alpha}^{\min \{u, r\}} \sum_{t=\beta}^{\min \{v, r\}} a^{(u-s) t} b^{(u-s)(r-s-t)} c^{(v-t)(r-s-t)} f_{s, t, r-s-t}^{r} f_{u-s, v-t, w+s+t-r}^{n-r}$,

where $\alpha=\max \{0, u+r-n\}$ and $\beta=\max \{0, v+r-n\}$. This formula yields the elements $c_{p q}(n, i, r)$, and hence gives the structure constants for the basis of the Koszul dual associated to the elements $f_{u, v, w}^{n}$ 's and a closed formula for the multiplication in the Hochschild cohomology ring of $\Lambda$.

\section{REFERENCES}

[1] Buchweitz, R.-O., Green, E. L., Madsen, D., Solberg, Ø., Finite Hochschild cohomology without finite global dimension, Math. Res. Lett., to appear.

[2] Cibils, C., Cohomology of incidence algebras and simplicial complexes, J. Pure Appl. Algebra 56 (1989), no. 3, 221-232.

[3] Cibils, C., Hochschild cohomology algebra of radical square zero algebras, Algebras and modules II (Geiranger, 1996), 93-101, CMS Conf. Proc., 24, Amer. Math. Soc., Providence, RI, 1998.

[4] Erdmann, K., Holloway, M., Snashall, N., Solberg, Ø., Taillefer, R., Support varieties for selfinjective algebras, K-Theory, vol. 33, no. 1 (2004), 67-87.

[5] Gerstenhaber, M., A uniform cohomology theory for algebras, Proc. Nat. Acad. Sci. U.S.A. 51 (1964), 626-629.

[6] Green, E. L., Huang, R. Q., Projective resolutions of straightening closed algebras generated by minors, Adv. Math. 110 (1995), no. 2, 314-333.

[7] Green, E. L., Hartman, G., Marcos, E. N., Solberg, Ø., Resolutions over Koszul algebras, Archiv der Mathematik, to appear.

[8] Green, E. L., Snashall, N., Solberg, Ø., The Hochschild cohomology ring modulo nilpotence of a monomial algebra, J. Algebra and its applications, to appear.

[9] Green, E. L., Solberg, Ø., An algorithmic approach to resolutions, to appear.

[10] Green, E. L., Solberg, Ø., Zacharia, D., Minimal projective resolutions, Trans. Amer. Math. Soc., 353 (2001), 2915-2939.

[11] Happel, D., Hochschild cohomology of finite-dimensional algebras, Séminaire d'Algèbre Paul Dubreil et Marie-Paul Malliavin, 39ème Année (Paris, 1987/1988), 108-126, Lecture Notes in Math., 1404, Springer, Berlin, 1989.

[12] Keller, B., Derived invariance of higher structures on the Hochschild complex, preprint.

[13] Martínez-Villa, R., Zacharia, D., Auslander-Reiten sequences, locally free sheaves and Chebysheff polynomials, Compos. Math., to appear.

[14] Priddy, S. B., Koszul resolutions, Trans. Amer. Math. Soc., 152 (1970), 39-60.

[15] Sanada, K., On the Hochschild cohomology of crossed products, Comm. Algebra 21 (1993), no. 8, 2727-2748.

[16] Snashall, N., Solberg, Ø., Support varieties and Hochschild cohomology rings, Proc. London Math. Soc., (3) 88 (2004), no. 3, 705-732.

[17] Siegel, S. F., Witherspoon, S. J., The Hochschild cohomology ring of a group algebra, Proc. London Math. Soc. (3) 79 (1999), no. 1, 131-157. 
Ragnar-Olaf Buchweitz, Department of Mathematics, University of Toronto, ToRonto, ON Canada M5S 3G3, Canada

E-mail address: ragnar@math.toronto.edu

Edward L. Green, Department of Mathematics, Virginia Tech, Blacksburg, VA 24061, USA

E-mail address: green@math.vt.edu

Nicole Snashall, Department of Mathematics, University of Leicester, University RoAd, Leicester, LE1 7RH, England

E-mail address: N.Snashall@mcs.le.ac.uk

Øyvind Solberg, Institutt for matematiske fag, NTNU, N-7491 Trondheim, Norway

E-mail address: oyvinso@math.ntnu.no 